\title{
Computer software and hardware to determine contrast sensitivity using three methods: Tracking, limits, and constant stimuli
}

\author{
DONALD J. HATCHER \\ Naval Aerospace Medical Research Laboratory, Pensacola, Florida \\ TERRY L. DEVIETTI \\ Central Washington University, Ellensburg, Washington \\ and \\ JOHN A. D'ANDREA \\ Naval Aerospace Medical Research Laboratory, Pensacola, Florida
}

\begin{abstract}
The software and hardware to obtain contrast sensitivity functions in a single session with monkeys are described. The versatility of the software allows the program to be used in a variety of sensory systems with only minor programming modifications. It will permit absolute or difference threshold measurements with any one of three methods of threshold testing: (1) method of tracking, (2) method of limits, and (3) method of constant stimuli. Human and monkey contrast sensitivity functions measured with the software and hardware are provided.
\end{abstract}

Contrast sensitivity functions (CSFs) are a fundamental description of spatial vision performance (Campbell \& Green, 1965). During the past decade, they have become a widely used means to assess spatial visual performance in both humans and animals. Basically, A CSF shows the capability to perceive a target from its surroundings under dissimilar luminance conditions. Traditionally, sine wave gratings presented at different contrasts have been used to determine CSFs. Contrast, as pertaining to contrast sensitivity, can be defined as the difference in luminance of the background and the target. Spatial frequency (cycles/degree) is determined by the size of the target, with small gratings representing a high spatial frequency and large gratings representing a low

This research was sponsored by the Naval Medical Research and Development Command under work unit 62758N MM 58524.0020010. The views expressed in this article are those of the authors and do not reflect the official policy or position of the Department of the Navy, Department of Defense, or the U.S. Government. Trade names of materials and/or products of commercial or nongovernmental organizations are cited as needed for precision. These citations do not constitute official endorsement or approval of the use of such commercial materials and/or products. The animals used in this work were handled in accordance with the principles outlined in the Guide for the Care and Use of Laboratory Animals, prepared by the Committee on Care and Use of Laboratory Animals of the Institute of Laboratory Animal Resources, National Research Council, DHHS, NIH Publication No. 86-23, 1985, and The Animal Welfare Act of 1966, as amended in 1970 and 1976. Correspondence should be addressed to the authors, Commanding Officer, Attention: Code 24, Dr. J. A. D'Andrea, NAVAEROMEDRSCHLAB, 51 Hovey Road, Pensacola, FL 32508-1046. spatial frequency. By conducting tests at several spatial frequencies, a CSF can be constructed to reflect the ability of the visual system to detect targets of different sizes and contrasts. While several studies have measured CSFs very effectively in rhesus monkeys (e.g., Boltz, Harwerth, \& Smith, 1979; De Valois \& Morgan, 1974; Jacobs, 1977; Williams, Boothe, Kiorpes, \& Teller, 1981), the program and hardware described in this report were more productive for our requirement to evaluate visual function in rhesus monkeys (Macaca mulatta) during 1.5-h test sessions. The program incorporates four levels of training to establish stable performance in nonhuman primates. In addition, the program provides online feedback to the experimenter, which is helpful during training stages and measurement of CSFs.

Several researchers (Blough 1958, 1966; Gourevitch, 1970; Rosenberger, 1970; Stebbins 1970) have established behavioral procedures to determine sensory thresholds in animals. Threshold determination procedures include (1) the method of tracking, (2) the method of limits, and (3) the method of constant stimuli. These procedures provided a basis for the development of our training procedure (DeVietti, D'Andrea, Hatcher, \& Reddix, 1993) and for the software and hardware presented here that was necessary to support the training procedure. In combination, these methods can be used to determine CSFs or other sensory thresholds using any one of the traditional threshold testing methods.

The following is an overview of the hardware and computer program developed to generate contrast displays on a high-resolution XYZ-axis display device (Tektronix 606B) and to measure human and monkey 
(Macaca mulatta) CSFs. The display consists of vertical sinusoidal gratings that can be computer controlled by the experimenter for varying degrees of contrast and spatial frequency. This program was developed to provide such visual stimuli to determine CSFs, train monkeys on the procedure, record and store data, and identify threshold contrast sensitivity levels.

\section{Hardware and Software Requirements}

Certain hardware components are required for the program to function and evaluate contrast sensitivities. Figure 1 shows a block diagram of the complete setup required for proper operation. An IBM-compatible microcomputer is used to run the program. To accurately measure the reaction time of responses, a Metrabyte CTM-5 timer is used. The timer card measures the amount of time it takes for the subject to make a left-lever response once the contrast has been displayed. A faster microcomputer, such as an $8048625-\mathrm{MHz}$, will ensure accurate response-time measurements. Reaction time obtained by the program with an $8048625-\mathrm{MHz}$ computer was calibrated by connecting the output of the function generator to channel one and the left response lever to channel two of a storage oscilloscope (Tektronix model 2230). Response time was measured from the time the grating was present until the left lever, indicating the stimulus had been detected, was activated. The time measured by the oscilloscope was compared with the time measured by the program and reported on the experimental printout. Average reaction times recorded by both techniques were within $3.5 \%$. Slower computers, for example, the 80286 , will suffice if accuracy to 2 or 3 hundredths of a second is not needed. A Tektronix XYZ monitor, Model 606B or compatible, is used to display the gratings. To produce the gratings, a rectangle-pulse generator and a ramp-wave generator are used to produce inputs to the $\mathrm{X}$ and $\mathrm{Y}$ inputs, respectively, of the $\mathrm{XYZ}$ monitor. A Metrabyte function generator Model PCIPSST is connected to the $\mathrm{Z}$ input to control the frequency and amplitude of the grating. In addition, a potentiometer is placed in series with the $\mathrm{Z}$ input of the monitor and the PCIP-SST to permit a wider range of amplitudes above and below the threshold level by effectively raising the zero voltage level to a positive voltage. All of the hardware components described here are required for correct operation of the TLC.EXE software program. Hardware substitutions could be made, but modification of the software program would be required.

\section{Program Operation}

The program, TLC.EXE, was written and compiled using the Microsoft BASIC Professional Development System 7.1 (Microsoft, 1987) and requires an IBM PCcompatible computer. A minimum disk space of $150 \mathrm{~K}$ is needed, and the program should be operated from a fixed disk. The program is "operator-input controlled," allowing the user to configure each experiment individually to the method of testing, length of test, duration of contrast presented, timeout (TO) period, spatial fre- quency, contrast value, variable interval (VI), and, if needed, catch trials. Certain variables, including VI, contrast intensity, TO, and spatial frequency, can be modified during the experiment by pressing the corresponding function key. The program is divided into four stages. Stages 1, 2, and 3 are training stages. Stage 4 determines the contrast sensitivity threshold. A startup menu allows the experimenter to choose one of the stages. The training stages are primarily designed to teach the subject how to perform the desired task. The function of Stage 1 is to familiarize the subject with the gratings and environmental surroundings. During this training, a contrast is always visible and reinforcements are supplied at VIs determined by the experimenter. The contrast can be displayed at fixed spatial frequencies and amplitudes, randomly selected spatial frequencies and amplitudes, or any combination of both.

Stage 2 introduces the subject to discrimination testing and is divided into two phases. Phase 1 of Stage 2 requires the subject to make a left-lever response to obtain reinforcement. As in Stage 1, spatial frequencies and amplitudes can be selected at random or remain at fixed values. Once the subject has mastered the reinforcement process, the stimulus is placed on a VI schedule (Phase 2). A left-lever response during a contrast produces a reinforcement, terminates the contrast, and selects the next VI. When the experimenter has determined that the subject is responding to the contrast, a second response control (right lever) is added. During this training process (Stage 3 ), a contrast is presented when the subject makes a right-lever response. Again, as in Phase 2, a left-lever response delivers a reinforcement, removes the contrast, and determines the next VI. After the subject learns the process of pulling the right lever to present a contrast followed by a left-lever response for reinforcement, the gratings are placed on a VI schedule.

Stage 4 is the actual contrast sensitivity testing procedure. From this stage, the experimenter can determine the contrast sensitivities of the desired spatial frequencies by any of the three methods mentioned above. If the tracking method is selected, the program will automatically adjust the contrast values determined by a previously presented contrast. If the subject responds correctly to a presentation, the subsequent contrast will decrease by one step $(0.06 \mathrm{log})$. When the subject fails to respond, the next contrast will increase by one step. This continues until the experimenter can determine a threshold based on whether the subject responds or does not respond to a contrast. Figure 2 is a flow chart for the tracking method used in the TLC.EXE program. Operating the program in the limits mode allows the user to increase or decrease the contrast manually. Threshold levels are determined by recording the values to which the subject did and did not respond. The last method, constant stimuli, allows the experimenter to dictate the amplitude values and the total number of times each contrast will be displayed. These values are selected at random until each contrast value has been displayed or the user terminates the test. 


\section{CONTRAST}

SENS I T I V I T Y

Block Diagram

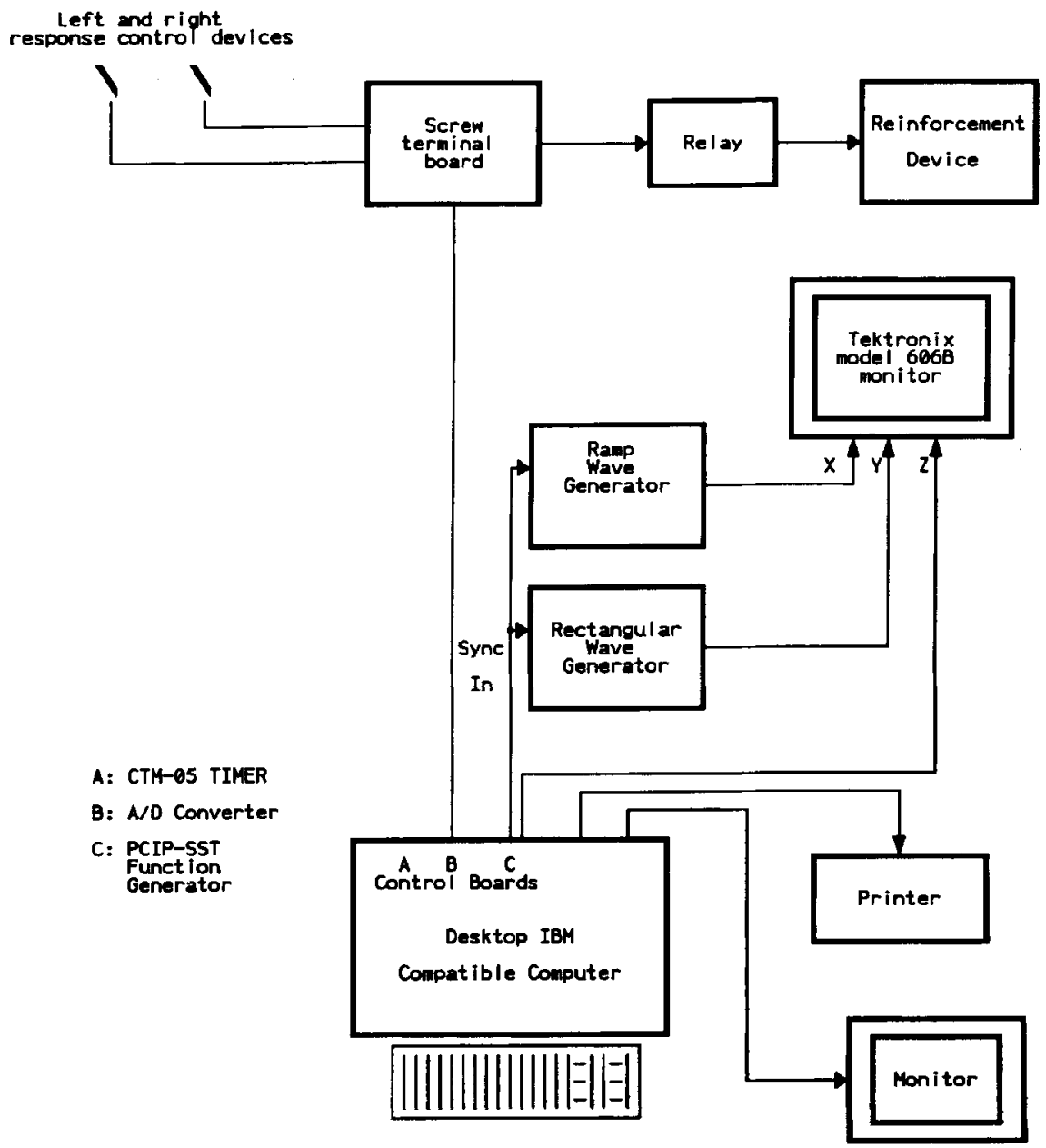

Figure 1. Block diagram of hardware.

The duration of a manually presented contrast is indefinite; the subject can terminate presentation by making a left-lever response, or the experimenter can terminate it by pressing the space bar on the computer keyboard. In both cases, a reinforcement is delivered, the contrast is removed, and a new VI is started. The sleep time allows the subject to continue activating the left lever and not be penalized with a timeout. During the sleep time, the next interval is selected, and the program continues normally. In all four stages, menu selections allow the user to input the file name, type of experiment (tracking, limits, constant stimuli), length of session, VI, TO, duration of stimulus, sleep time, catch trials, spatial frequency, and contrast amplitude. The VI is the average of all the variable intervals presented. Entering a VI of 20 would mean that the experimenter wants a contrast to be presented on an average of every $20 \mathrm{sec}$. The intervals for each VI were selected using the formula developed by Fleshler and Hoffman (1962). The length of a TO is entered in seconds. Each time the subject makes an incorrect response (left-lever response when a contrast is not being presented), the program automatically sends a $1-\mathrm{Hz} 0-\mathrm{V}$ signal to the monitor, causing the display to flash on and off. This TO period is used to reduce the amount of "guessing" by the subject that may occur at or near threshold levels. The duration of the stimulus is the length of time the contrast is displayed on the monitor. While the program is running, the experimenter can manually present a stimulus or deliver an immediate reinforcement to the subject.

To conclude that artifacts are not affecting subject responses, catch trials $(.01 \mathrm{~V} \mathrm{dc})$ may be entered into the random-interval selection process. These catch trials are presented exactly as a regular test grating and can help 

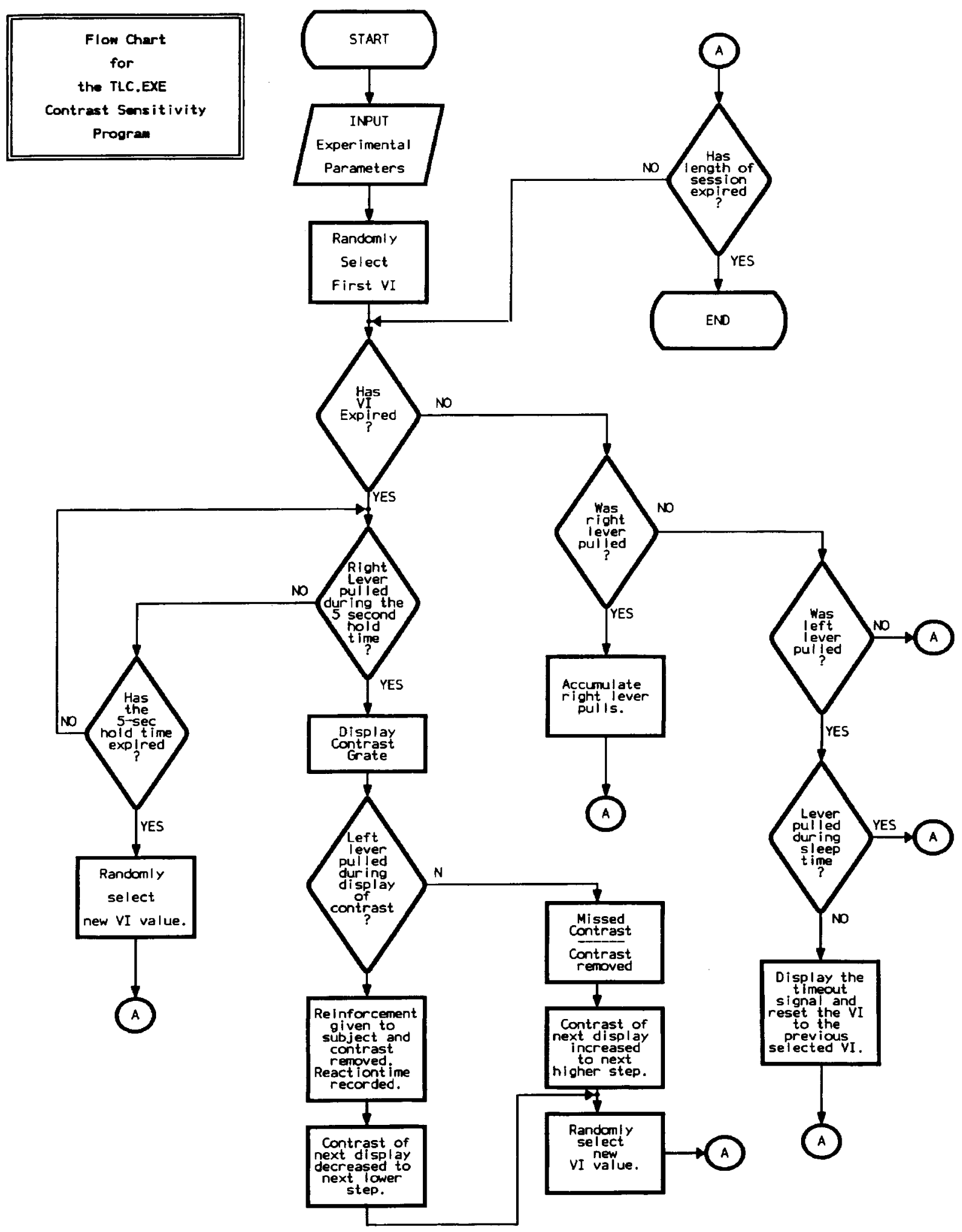

Figure 2. Flow chart of tracking method. 
determine if any outside interference, such as noise, electromagnetic interference, or flicker, are causing the subject to respond to the grating.

Spatial frequencies are determined by the distance of the eye to the target, size of the target, and the number of gratings displayed on the monitor. The number entered for a specific spatial frequency is the actual frequency of the PCIP-SST frequency generator that the monitor requires to display a given spatial frequency. This will vary depending on the type of equipment being used and desired spatial frequencies. Data lines in the BASIC programming source code contain the frequency values required to display spatial frequencies of $1.5,3.0$, $6.0,12.0$, and 18.0 cycles/deg of visual angle, with a target diameter of $3 \mathrm{~cm}$ and a distance from the subject of $100 \mathrm{~cm}$ using the hardware described previously.

To select the starting amplitude when using the tracking method, the experimenter enters a value that has been determined to be above the threshold level of the subject. Once a response is made to the grating, the next lower value is selected by the program. The program uses contrast values between 0.2542 and 0.0035 in 0.06 log steps, which extend well above and below the normal threshold levels in both humans (Vistech, 1988) and macaques (e.g., De Valois \& Morgan, 1974).

\section{Experimental Feedback: Screen, Printer, and Tones}

The feedback that the experimenter receives from the computer screen is shown in Figure 3 and is useful in determining certain statistics concerning the subject during the testing procedure. The PCIP-SST function generator is programmed to show the current output values being sent to the Tektronix 606B monitor (top portion of Figure 3). Frequency, amplitude, type of signal, and the dc offset value are displayed and can be changed manually at any time during the experiment. The program updates every trial and displays the results on the computer screen. These data include the start time of the test session, current session time, current VI value and the remaining time until the VI expires, length of TOs, number of TOs, right-lever pulls with and without a contrast, and the total number of reinforcements received. In addition, the method of testing, spatial frequency, amplitude, and the average of all the VIs selected are displayed.

Output to a printer is updated following each trial, showing the experimental method, reaction time, contrast value, spatial frequency, and a graph illustrating the progress from trial to trial (see Figure 4). At the end of the experiment, the program compiles the data, performs all necessary calculations, and prints the results. When using the tracking method, the total number of trials and the number of trials that were responded to correctly at each contrast are printed. A percentage of correct to incorrect responses is also indicated. All data are recorded to an ASCII file for further evaluation and future reference.

Audible tones are produced at the computer to indicate certain events, such as right-lever and left-lever pulls, TOs, catch trials, and the expiration of a VI. By discerning the meaning of each tone, the experimenter can readily acknowledge the current status of the test. Not only does this feedback return the activity of the subject, it also provides the user with some assurance that the program is functioning properly.

Software requirements include the TLC.EXE program and the drivers needed to operate the PCIP-SST function generator. These PCIP-SST drivers come with the function generator and are loaded manually or auto-
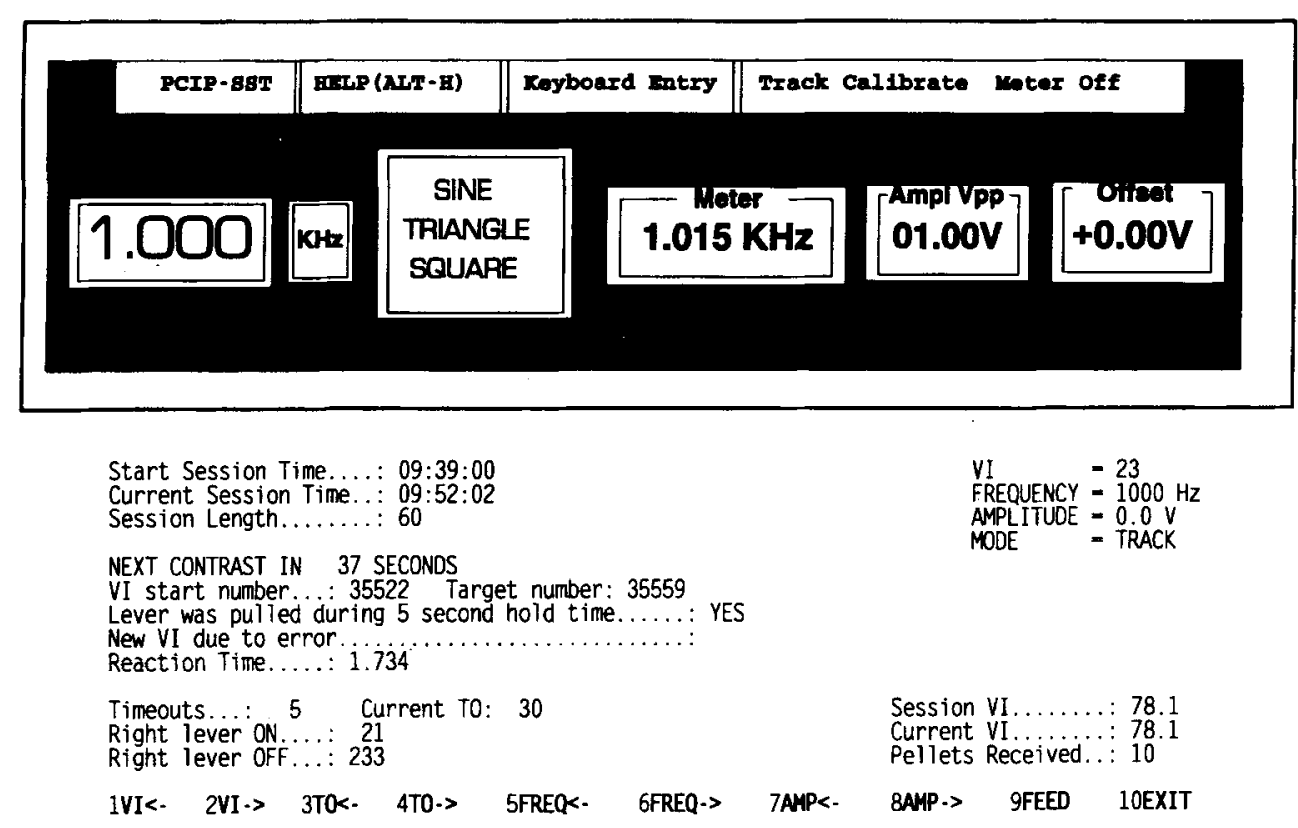

Figure 3. Ilustration of on-line program feedback to the experimenter. 
matically in the CONFIG.SYS file during the boot-up procedure of the computer. This software also allows the user to generate frequencies and amplitudes outside the TLC.EXE program, making it readily accessible for troubleshooting. If changes are made to the source code, the program must be recompiled. A library, CTM5COM.LIB, containing the commands for the CTM-5 timer is supplied with the program. This library must be loaded from the command line (QBX /L CTM5COM.LIB) before the TLC.EXE file can be created.

\section{Testing of the Hardware and Software}

Four monkeys (Macaca mulatta) were trained using the hardware and software described here and provided CSFs at spatial frequencies ranging from 1.5 to 18 cycles/deg (DeVietti et al., 1993). The monkeys were seated in a polyvinyl chloride (PVC) pipe chair for approximately $1.2 \mathrm{~h}$ each session. A pellet feeder (Foringer $1 \mathrm{~g}$ ) mounted outside the chamber delivered food pellets to the monkey chair through a $1.6-\mathrm{m}$ length of Tygon tubing. Each monkey wore a plastic collar and was properly acclimated to the pole-and-collar handling procedure (Anderson \& Houghton, 1983). The chair had two plastic levers ( $7 \mathrm{~cm}$ long, $1.3 \mathrm{~cm}$ diam) mounted vertically and in front of the animal: one to the right and the other to the left. Fiber-optic light switches (Microswitch CJWZ-3IIP-B) were activated when the monkey pulled the levers. The fiber-optic switches were connected to light-emitting diodes and light detectors outside the chamber (Microswitch No. FE7C-FR6M) with 15.2-m lengths of fiber-optic cable.

The monkeys were placed in a rectangular anechoic chamber. The chamber was constructed of wood framework with the interior lined by sheet metal covered with a sound-attenuating absorber. A white-noise source (AM-1100, Soundolier Inc.) at floor level produced a 75-dBA masking sound inside the chamber at the location of the chair. Output of the white noise source was evaluated in one-third octave bands with a spectrum analyzer (RT Acoustics, Computational Systems Inc.). The majority of the energy was in the frequency range of $125-3150 \mathrm{~Hz}$, with a rapid falloff between 2000 and $5000 \mathrm{~Hz}$. An axial fan ventilated the chamber $\left(7 \mathrm{~m}^{3}\right.$ per minute), and two spotlights ( $75 \mathrm{~W}$ ) illuminated the interior. An additional spotlight was mounted at the rear of the chamber to illuminate the monkey chair for safe chairing of the monkeys, but was turned off and covered with absorber material when not in use during behavioral test sessions. We monitored the subjects' behavior by a television camera and monitor (Hitachi FP-7). A video recorder (Panasonic Model No. AG-6400) was used to record monkey behavior during exposures. An analog thermometer (Yellow Springs No. 43TB) was used to monitor ambient air temperature of the chamber.

The monkeys were purchased from Charles River Laboratories, Wilmington, MA, and were certified Simian Herpes B virus negative. The mean weight of the subjects during the study was $5.63 \mathrm{~kg}( \pm 0.14 \mathrm{~kg} S E M)$. The subjects were fed a standard primate diet (Purina Co., $25 \%$ protein) daily in sufficient quantities (freely available in their cages) to produce a normal-sized animal for that age. During the experiment, the animals obtained their daily food ration (BioServ, Holton Industries Co., 1-g monkey banana formula dustless pellets) while performing the experiment. Their diet during the experiment was supplemented with fresh fruit. The animals were individually housed in an American Association for the Accreditation of Laboratory Animal Care (AAALAC) accredited facility. Photoperiod was regulated to 12-h light and 12-h dark (0630 on, 1830 off). Home cage temperature was maintained at an average $22.6^{\circ} \mathrm{C}\left( \pm 0.11^{\circ} \mathrm{C} \mathrm{SEM}\right)$.

Luminance measurements on the CRT were made with a Pritchard Photometer (Model PR-1980A). The luminance of the CRT was set for an overall luminance of $24 \mathrm{~cd} / \mathrm{m}^{2}$ during all contrast presentations as well as in the absence of a contrast. Thresholds were evaluated using the tracking procedure. A contrast value above threshold was selected, and the monkey's response to this contrast determined the contrast value of the next trial. A correct response to the contrast decreased the contrast level one unit, whereas an incorrect response to the contrast increased the contrast level one unit. In this manner, the monkeys determined a threshold, and their performance oscillated about the threshold for that particular spatial frequency. The order of the presentation of the five spatial frequencies was randomized for each day.

To determine that the system is operating correctly, the experimenters often perform the task and establish their own CSF. They were tested in a single session with the same spatial frequencies using the tracking procedure under the conditions as close as possible to that in which the monkeys performed. The human volunteers, in strict compliance with laboratory protocol, wore gloves, masks, and protective clothing. They knelt or sat behind the monkey chair in the chamber and leaned over the chair to place their eyes in the correct position and distance from the CRT. Like the monkeys, these volunteers responded with the right lever to produce a contrast on a VI schedule and pulled the left lever to indicate the perception of a contrast. The auditory cues of the feeder provided feedback for correct performance. Additional details of the training procedure can be found elsewhere (DeVietti et al., 1993).

Typical CSFs for one monkey (age 3 years) and human (age 55 years) are shown in Figure 5 . The threshold CS for each spatial frequency is plotted and a second order regression fitted to each data set using a commercial plotting program (SigmaPlot, Ver. 5.0, Jandel Scientific). The CSFs appear to fit very well with other sources of human and monkey data (Harwerth \& Smith, 1985). To evaluate the sensitivity of this procedure and system, CSFs were established for one monkey viewing the contrast gratings through trial lenses with powers of $0.75,1.25$, and 2.25 diopters. A flat plastic plate was mounted to the chair and used to hold the lenses, and the monkeys were required to view the CRT through 
File - a1271620.tr2

STAGE 3

ab-10-1972

Q6istints

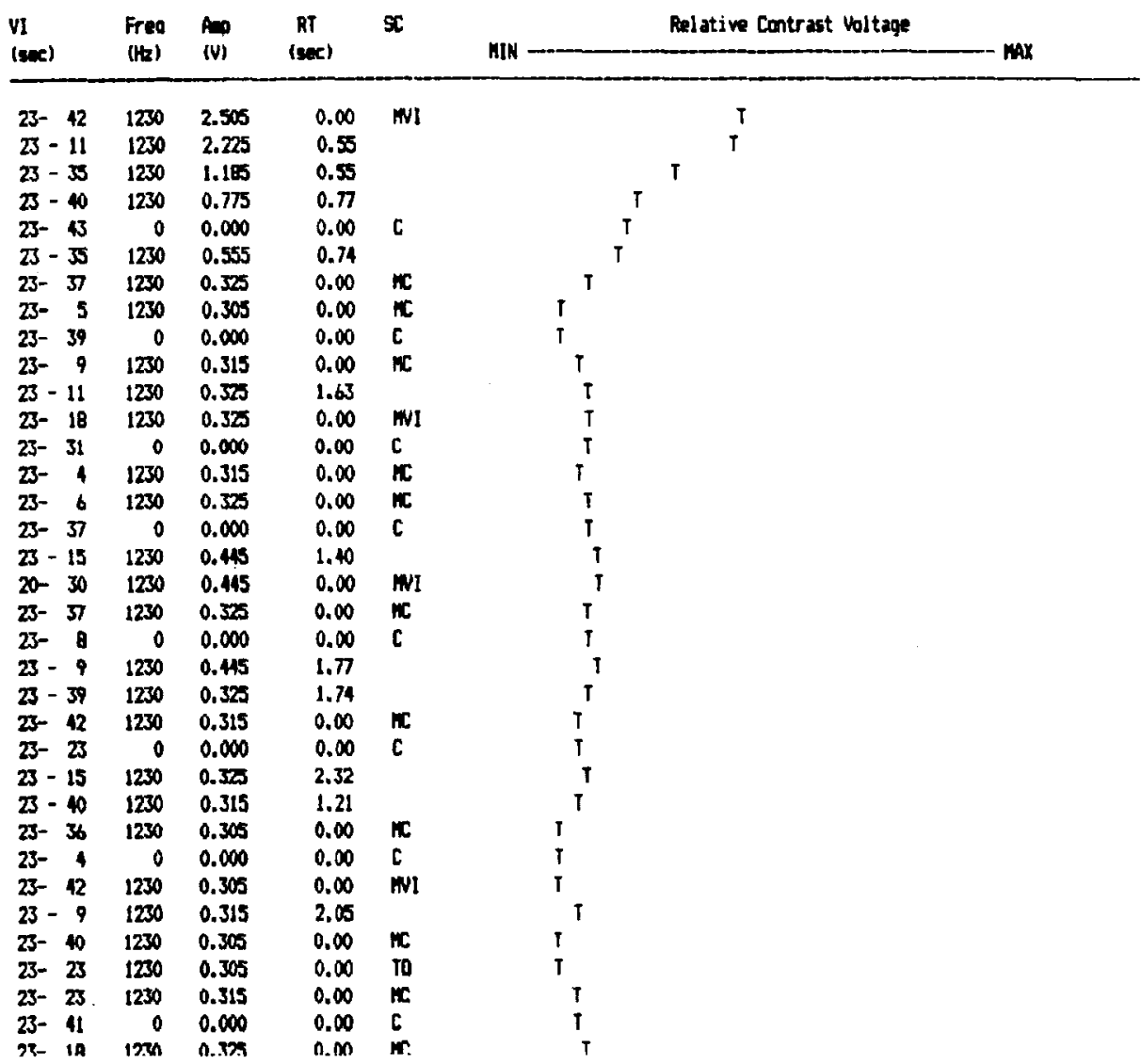

Froquency tested 1230 th

Contrast Sensitivity Voltage No. Presented Correct Responses
/ Correct

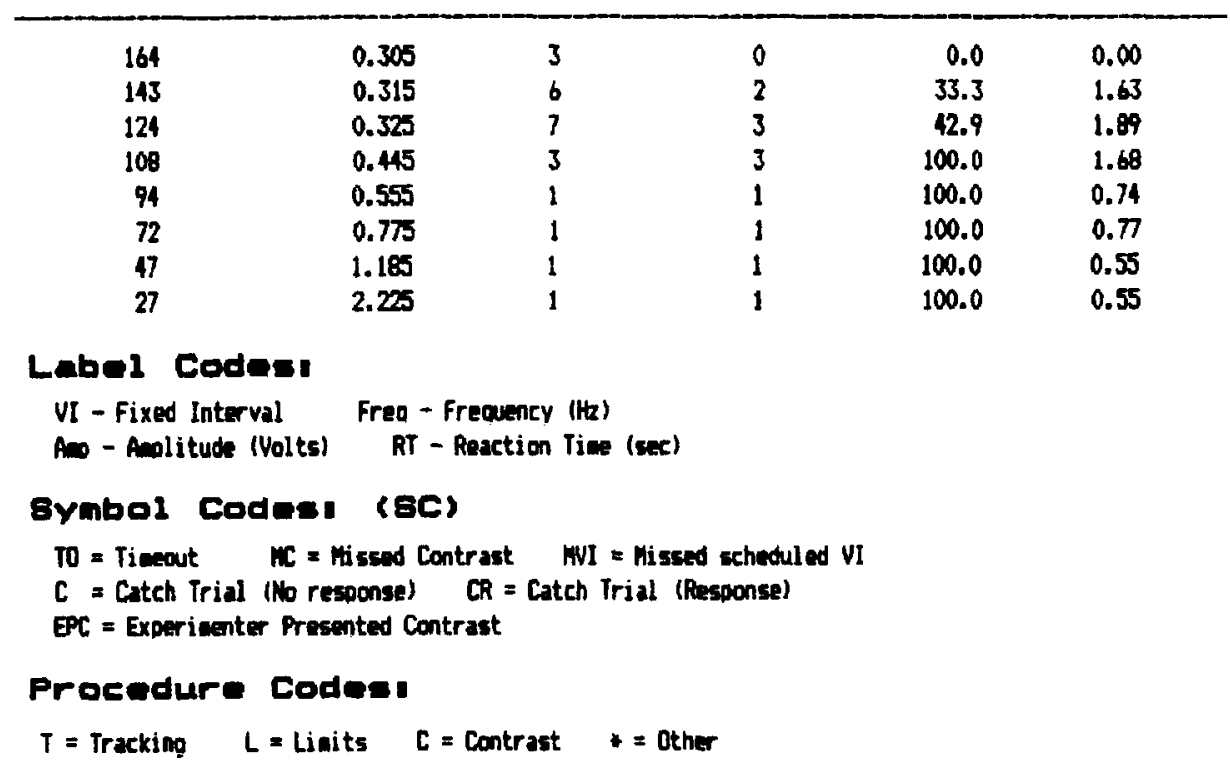

Figure 4. Real-time computer printout of an actual experimental test. 
the lenses. It was constructed so that trial lenses could be easily inserted and removed. As is shown in Figure 6, CSFs were altered dramatically by the trial lenses, with greater effects seen at the highest power lens. The primary change occurred at the high spatial frequencies, which is expected since the grating on the CRT is increasingly defocused by the higher lens powers.

\section{Summary}

We developed a computer program (TLC.EXE) with supporting hardware and training procedure (DeVietti et al., 1993) to determine contrast sensitivity functions in the monkey within a single session. By utilizing human subjects to determine the operational integrity of the program, we have also demonstrated the ability to perform CSF testing on humans in the same manner as ascertaining CSFs for the monkey (Figure 5).

The program, with supporting hardware described in this paper, controls all events and allows threshold testing by any one of three procedures (the method of constant stimuli, the method of limits, and the method of tracking). The program also provides both detailed online event-by-event monitoring and session summary results and allows within-session changes in the procedure. Although the program was written initially to perform tests for contrast sensitivity, it can be used readily, with minor modification, to determine other sensory thresholds. It has been reconstructed in our laboratory to conduct hearing threshold experiments (AUDIO.EXE) in macaques. The output of the PCIP-SST function generator is used to change the frequency and amplitude of a signal connected to a speaker. Responses are made to

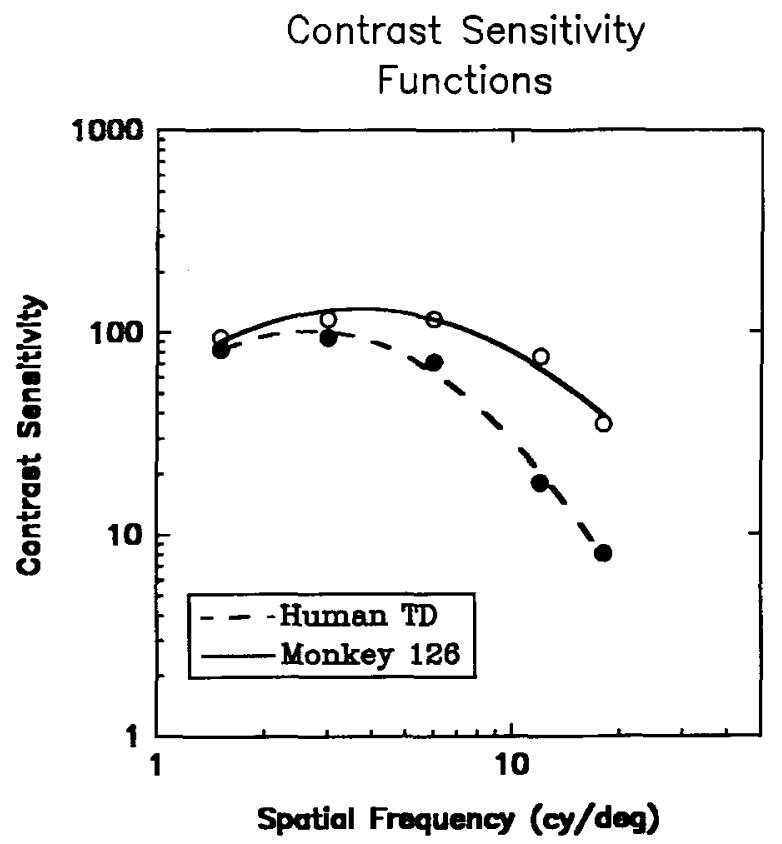

Figure 5. Contrast sensitivity functions determined for a monkey and a human using the tracking procedure.

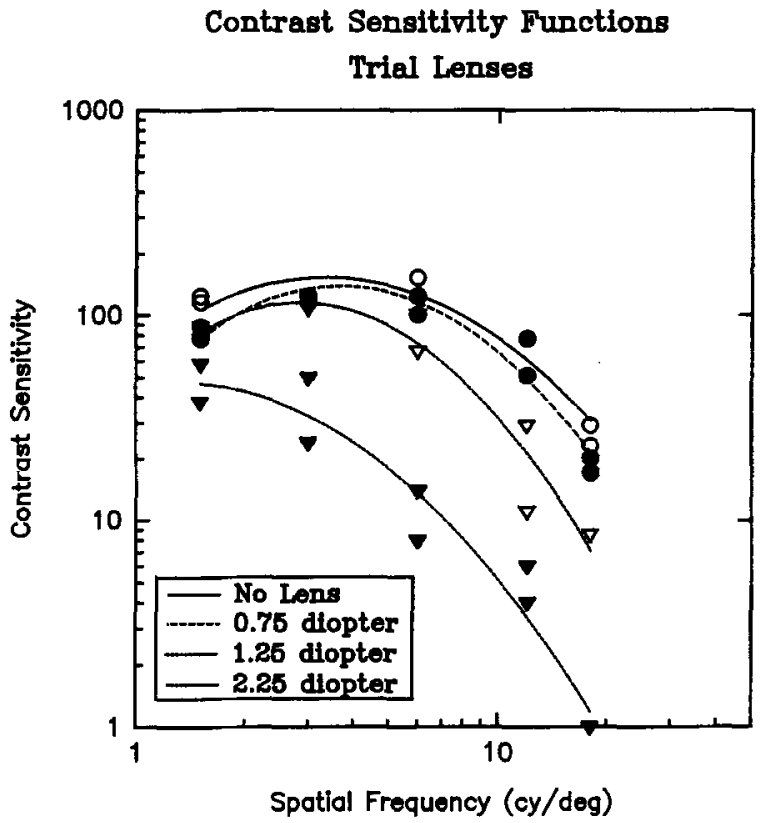

Figure 6. Contrast sensitivity functions for a monkey viewing the CRT through trial lenses of different optical powers.

a tone instead of a grating. Tracking is accomplished by decreasing or increasing the sound pressure level $(\mathrm{dB})$ as the subject correctly or incorrectly responds to the tone. Because of the versatility of the TLC.EXE program, other sensory threshold experiments that utilize a sine, square, or triangle waveform from the function generator to present a stimulus to the subject can be readily accomplished. The program could support hardware similar to that described in this paper with appropriate modifications to the TLC.EXE source code.

\section{Availability}

To receive a free copy of the software, send a selfaddressed stamped container with either a 3.5 -in. or a 5.25-in. IBM-compatible formatted disk to the authors.

\section{REFERENCES}

Anderson, J. H., \& Houghton, P. (1983). The pole and collar system: A technique for handling and training nonhuman primates. Lab Animal, 12, 47.49.

BLOUGH, D. S. (1958). A method for obtaining psychophysical thresholds from the pigeon. Journal of the Experimental Analysis of Behavior, 1, 31-43.

Blough, D. S. (1966). The study of animal sensory processes by operant methods. In W. K. Honig (Ed.), Operant behavior: Areas of research and application (pp. 345-379). New York: AppletonCentury-Crofts.

Boltz, R. L., HaRWerth, R. S., \& SMith, E. L. (1979). Orientation anisotropy of visual stimuli in rhesus monkey: A behavioral study. Science, 205, 511-513.

CAMPBell, F., \& GreEN, D. (1965). Optical and retinal factors affecting visual resolution. Journal of Physiology, 181, 576-593.

DE Valois, R. L., \& Morgan, H. (1974). Psychophysical studies of monkey vision: III. Spatial luminance contrast sensitivity tests of macaque and human observers. Vision Research, 14, 75-81.

DeVietti, T. L., D'Andrea, J. A., Hatcher, D. J., \& Reddix, M. D. 
(1993). A training procedure for obtaining contrast sensitivity functions within a single session in monkeys. Bulletin of the Psychonomic Society, 31, 245-248.

FLESHLER, M., \& HoFfMAN, H. S. (1962). A progression for generating variable-interval schedules. Journal of the Experimental Analysis of Behavior, 5, 529-530.

Gourevitch, G. (1970). Detectability of tones in quiet and in noise by rats and monkeys. In W. C. Stebbins (Ed.), Animal psychophysics (pp. 67-97). New York: Plenum Press.

HARWERTH, R. S., \& SMITH, E. L. (1985). Rhesus monkey as a model for normal vision of humans. American Journal of Optometry \& Physiological Optics, 62, 633-641.

JACOBS, G. H. (1977). Visual capacities of the owl monkey (Aotus trivirgatus): II. Spatial contrast sensitivity. Vision Research, 17, 821-825.

Microsoft CoRporation (1989). Microsoft BASIC professional de- velopment system [Computer program]. Bothel, WA: Author. ROSENBERGER, P. B. (1970). Response-adjusting stimulus intensity. In W. C. Stebbins, (Ed.), Animal psychophysics (pp. 161-184). New York: Plenum Press.

Stebirns, W. C. (1970). Studies of hearing and hearing loss in the monkey. In W. C. Stebbins (Ed.), Animal psychophysics (pp. 41-66). New York: Plenum Press.

VISTECH CONSUlTANTS, INC. (1988). Instruction manual. Dayton, $\mathrm{OH}$ : Vistech Consultants, Inc.

Williams, R. A., Boothe, R. G., Kiorpes, L., \& Teller, D. A. (1981). Oblique effects in normally reared monkeys (Macaca nemestrina): Meridional variations in contrast sensitivity measured with operant techniques. Vision Research, 21, 1253-1266.

(Manuscript received February 9, 1993; revision accepted for publication April 18, 1994.) 